\title{
Impact of p21 Knockout on Topotecan-Induced Stress Responses in Human Colon Carcinoma Cells: A Proteomic Analysis
}

\author{
Kyunghee Lee ${ }^{1, \S}$ and Sayed S. Daoud ${ }^{1,2, *}$
}

\begin{abstract}
${ }^{I}$ Department of Pharmaceutical Sciences; ${ }^{2}$ Center for Integrated Biotechnology, Washington State University, Pullman, WA 99164-6534, USA and ${ }^{\S}$ Current Address: Division of Applied Life Science (BK21 Program) Gyeongsang National University, Gazwa-dong, Jinju, Gyeongnam, 660-701, Republic of Korea
\end{abstract}

\begin{abstract}
There are few reports describing the role of p21-dependent protein repression in cell death. To identify such cell death-associated proteins and to shed the light into the molecular mechanisms by which p21 is responding to pharmacological stress, we used a subcellular proteomic approach for the analysis of protein expression profiles of fractionated nuclei, mitochondria, and cytosols of isogenic p21 null (p21-/-) and wild-type human HCT-116 cells following treatment with sublethal doses $(1 \mu \mathrm{M})$ of the topoisomerase I inhibitor, topotecan (TPT). In total, 174 unique deregulated proteins were identified in HCT-116 cells following treatment with TPT, whereas only 146 proteins were identified in p21-/- cells. They contributed to multiple functional activities of stress signaling pathways, and that p21-/- cells are accelerated to be more responsive to topotecan-induced cell death due to the following: 1) down regulation of proteins involved in the transcriptional and replication machinery of cells like DNA (cytosine-5)-methyltransferase 1, Matrin 3, DNA replication licensing factor MCM4, heterogeneous nuclear ribonucleoprotein $\mathrm{Q}$, poly(Rc)-binding protein 1 and splicing factor arginine serine rich $7 ; 2$ ) the activation of a caspase-independent apoptosis by the upregulation of the Bcl 2 inhibitor of transcription (Bit1) protein; and 3) the activation of TNF $\alpha$ signaling by the upregulation of macrophage immigration inhibitory factor (MIF), and 26S proteosome non-ATPase regulatory subunit 2 (TRAP2) proteins. We suggest that the upregulation of these proteins are contributing factors to the molecular mechanisms of topotecan-induced cell death in p21-/- cells; and that the data present an opportunity for developing new therapeutic approaches for selective targeting of p21signaling pathways.
\end{abstract}

Key Words: Proteomics, topotecan, p21, colon cancer, mass spectrometry, Bit1.

\section{INTRODUCTION}

The eukaryotic DNA-damage response (DDR) has evolved to optimize cell survival following damage to the genome. Key DDR regulators are the phosphatidylinositol 3kinase-like kinases (PIKKs) ataxia telangiectasia mutated (ATM), ATM and Rad3 related (ATR), and DNA-dependent protein kinase catalytic subunit (DNA-PKcs), which are activated following DNA damage and then phosphorylate downstream targets [1-3]. Targets for ATM and ATR include transcription factor p53 and the checkpoint kinases CHK1 and CHK2; these in turn regulate the activities of downstream effort proteins controlling DNA repair, cell cycle progression, or the initiation of apoptotic or senescence programs.

In addition to being an important target of ATM and ATR, p53 is the most frequently mutated protein known in human cancers; with loss of p53 function thought to contribute to tumorigenesis by fostering genome instability and the consequent acquisition of additional oncogenic mutations $[4,5]$. One of the major properties of p53 is that its level and transcriptional activity are markedly induced by DNA damage and a range of other cellular stresses. Under normal con-

*Address correspondence to this author at the Department of Pharmaceutical Sciences, Washington State University, 340D Wegner Hall, Pullman, WA 99164-6534, USA; Email: daoud@wsu.edu ditions, p53 activity is maintained at low, basal levels through the actions of HDM2 (originally MDM2). HDM2 acts as a ubiquitin E3 ligase to transfer ubiquitin moieties into $\mathrm{p} 53$, thus promoting $\mathrm{p} 53$ degradation via the ubiquitindependent proteasomal system [6]. In response to DNA damage, negative regulation of p53 by HDM2 is lifted, leading to stabilization of transcriptionally component $\mathrm{p} 53$. This control is brought about in part by ATM- and ATR-mediated phosphorylation of both p53 and HDM2, which impairs the interaction between the two proteins [7].

A major consequence of p53 activation following DNA damage is the induction of cell-cycle arrest at the G1/S or G2/M transition stages. This is achieved primarily through p53-induced expression of target genes that encode factors such as $\mathrm{p} 21^{\mathrm{WAF} / \mathrm{CIP} 1}$, a negative regulator of cyclindependent kinases (CDKs) that induces G1/S arrest [8]. p21 has also been found to be involved in a variety of other cellular events, such as proliferation [9], differentiation [10], senescence [11], cell motility and tumor metastasis [12] as well as cell survival [13]. As for cell death (apoptosis), many conflicting data are published regarding the role of p21 in this active process. Cells lacking p21 function were shown to undergo normal p53-dependent apoptosis [14]. In contrast, p21 was found to protect cells from apoptosis [15]. Furthermore, p21 has differential effects on the sensitivity of cancer cells to ionizing radiation and chemotherapeutic agents. For 
example, an increased level of p21 was shown to enhance sensitivity of hepatoma and osteosarcoma cells to cisplatin [16]. In contrast, in colon cancer and embryonic fibroblast cells, increased sensitivity to chemotherapeutic agents is associated with the lack of p21 [17,18]. Thus, there is a need for further studies to understand the molecular stress responses of p21 following DNA damage in cells.

The aim of the study was to analyze the role of p21 in stress-induced responses after DNA damage using a subcellular mass spectrometry-based expression proteomic approach. We compared protein expression changes in the subcellular fractions of the human colon carcinoma cell line, HCT-116, with those in an isogenic p21 knockout (HCT-116 p21-/-, Ref. 18) after treatment with the topoisomerase I inhibitor topotecan (TPT). Topotecan (Hycamtin), a semi-synthetic water-soluble derivative of camptothecin, is a clinically useful agent. It is approved for first-line therapy of cisplatin-refractory ovarian cancer and second-line therapy of small lung cancer [19]. Like other camptothecins, topotecan converts topoisomerase I into a cellular poison by trapping topoisomerase I in a covalent complex with DNA. The cytotoxic lesions result from breaks, generated by collision of the complexes with DNA or RNA polymerase [20]. We analyzed protein expression profiles of fractionated nuclei, mitochondria and cytosols of TPT-induced stress responses, compared to controls, in HCT-116 and HCT-116 p21-/- cells by one-dimensional (1D) sodium dodecyl sulfate-polyacrylamide gel electrophoresis (SDS-PAGE) coupled with liquid chromatography electrospray-ionization-tandem mass spectrometry (LC/ESI-MS/MS) tandem mass spectrometry) [21,22]. In total, 174 unique deregulated proteins were identified in HCT-116 subcellular fractions after TPT treatment, whereas only 146 total proteins were identified in HCT-116 p21-/- subcellular fractions. These observations indicate that more proteins are induced in response to TPT treatment in the presence of functional p21 protein. The identified proteins contributed to multiple biological activities such as cell cycle, apoptosis and oxidative stress, protein metabolism and modification, cell structure and motility, nuclei acid metabolism, signal transduction and energy. The expression of some of these proteins in each cellular compartment, and their significance in stress responses and apoptosis was discussed.

\section{MATERIALS AND METHODOLOGY}

\section{Drugs and Materials}

Topotecan was obtained from the Developmental Therapeutics Program, National Cancer Institute, NIH. Stocks were prepared in $100 \%$ dimethyl sulfoxide (DMSO) and stored in aliquots at $-80^{\circ} \mathrm{C}$. Primary antibody Bit1 was purchased from ProSci (Poway, CA), and actin from Sigma (St. Louis, MO). The goat anti-rabbit and the donkey anti-goat secondary antibodies labeled with IRDye ${ }^{\mathrm{TM}} 38$ were purchased from LI-COR, Inc. Biosciences (Lincoln, NE) or with Alex680 from Molecular Probes, Inc. (Eugene, OR). All other chemicals were of reagent grade.

\section{Cell Culture, Drug Treatment and Cytotoxicity}

Isogenic p21-null (HCT-116 p21-/-) and wild-type (HCT-116) human colon carcinoma cells, kindly provided by Dr. Bert Vogelstein [18] were grown as monolayers in DMEM (Life Technologies, Inc., Gaithersburg, MD) sup- plemented with $10 \%$ fetal bovine serum (Hyclone, Logan, UT) as previously reported [23]. For cytotoxicity assays, cells from exponentially growing cultures were plated in 48well tissue culture plates in DMEM medium plus $10 \%$ fetal bovine serum at about $5 \times 10^{4}$ cells per well for overnight. Cells were treated with graded doses of TPT for 24 hours. Each treatment was preformed in quadruplicate wells in two independent experiments. The wells were washed twice with pre-warmed medium, followed by the addition of $1.5 \mathrm{ml}$ of drug-free medium. The plates were then incubated for a further 3 days. The surviving fraction of cells was determined with the crystal violet assay, as previously reported [21]. The precision of this method with quadruplicate determinations is $<10 \%$ (SD). The data were analyzed with Student's twotailed $t$-test; $P<0.01$ was considered statistically significant. For protein expression profiling, exponentially growing cultures at $80 \%$ confluence in $150 \mathrm{~cm}^{3}$ tissue culture flasks were treated for 24 hours with $1 \mu \mathrm{M}$ TPT. Control cells were treated with an equivalent volume of DMSO. Cells were washed with pre-warmed media, and harvested.

\section{Protein Extraction and Separation}

Harvested cells were subjected to subcellular (nuclear, mitochondrial, cytosolic) fractionation with the FOCUS ${ }^{\mathrm{TM}}$ protein extraction kit (Geno Technology, Inc., St. Louis, MO) in accordance with the manufacturer's instructions, as previously published by our laboratory [22]. The harvested cells were pelleted by centrifugation at $800 \mathrm{x} g$ for $1 \mathrm{~min}$. Cell lysis was performed using a narrow opening (20 gauge) syringe needle on ice, gently pulling cell suspension up and down 10-30 times. The resultant high purity (>95\%) of mitochondrial fractions was validated by a transmission electronmicroscopy (TEM) (data not shown). Protein concentrations were determined with $\mathrm{CB}-\mathrm{X}^{\mathrm{TM}}$ Protein Assay kit (Geno Technology Inc., St. Louis, MO). An equal amount of each protein sample $(30 \mu \mathrm{g})$ was separated by SDS-PAGE (10$20 \%$ ). Gels were stained with Coomassie Brilliant Blue G250 (BioRad, Hercules, CA)

\section{In-Gel Enzymatic Digestion and Mass Spectrometry}

Complete lanes of $1 \mathrm{~cm}$ each were cut into 18 pieces, and the protein bands were digested with trypsin (Promega, Madison, WI) as previously reported [30]. For tryptic peptide separation a Waters Nanoacquity Ultra Performance LC (UPLC) was used. In-gel digested $2 \mu 1$ samples were loaded into fused silica capillary Atlantis ${ }^{\mathrm{TM}} \mathrm{dC} 18,3 \mu \mathrm{m}, 75 \mu \mathrm{m} \mathrm{x}$ $100 \mathrm{~mm}$ nanoease-column in tandem with a pre-column; symmetry $\mathrm{C} 18,5 \mu \mathrm{m}, 180 \mu \mathrm{m} \times 20 \mathrm{~mm}$. The peptide mixture was separated using the following conditions: trapping for 3 min using $100 \%$ solution A $(100 \%$ water with $0.1 \%$ formic acid) at flow rate of $5 \mu \mathrm{l} / \mathrm{min}$. Separation at $0.25 \mu \mathrm{l} / \mathrm{min}$ with following solvent gradients; Isocratic $97 \%$ solution $\mathrm{A}$ and $3 \%$ mobile phase solution B $(100 \%$ ACN with $0.1 \%$ formic acid) for $1 \mathrm{~min}$; Gradient $60 \%$ solution $\mathrm{A}$ and $40 \%$ solution B for 90 min; Gradient $10 \%$ solution A and $90 \%$ solution B for $10 \mathrm{~min}$; Isocratic $10 \%$ solution $\mathrm{A}$ and $90 \%$ solution $\mathrm{B}$ for 10 min followed by gradient $97 \%$ solution $\mathrm{A}$ and $3 \%$ solution $\mathrm{B}$ in $1 \mathrm{~min}$. Atlantis column effluent was delivered directly to the Q-TOF Premier ${ }^{\mathrm{TM}}$ (Waters Corporation, Milford, MA) equipped with nanospray ion source. The mass spectra were acquired using the MassLynx Windows XP software, version 4.0 in the positive ion reflector mode. For data 
analysis, ProteinLynx Global Server 2.2 and Protein Expression Informatics System software version 1.0 were used for MS/MS mass spectra analysis, peptide-sequencing and protein identification. We also used Mass-Lynx's 4.0 Protein Sequencing modules for manual sequencing to confirm results obtained with ProteinLynx. After mass spectral data acquisition, the peak $\mathrm{m} / \mathrm{z}$ values were extracted and used to search the SwissPort sequence database (ftp://ftp.expasy.ch/ databases/sp_nrdb/sport.fas.gz), as previously reported [21, 22]. The assignments by ProteinLynx were further confirmed using the Mascot software (Matrix Science, London, UK) and/or manual interpretation.

\section{Database Searching}

Using the combination of LC-MS/MS database searching, a number of proteins were identified in each subcellular fraction. The protein identification was performed with ProteinLynx Global Server (Waters) with SwissPort database and the MASCOT MS/MS ion search software (http://www. matrixscience.com). The maximum number of missed tryptic cleavages per peptide was set at one. Carbamidomethylation of cysteine and oxidation of methionine were allowed as fixed and variable modifications, respectively. Searches specified tryptic digestion used a peptide mass tolerance of $100 \mathrm{ppm}$ and a fragment ion tolerance of 0.1 Da. Peptide identifications were accepted if they could be established at greater than $95 \%$ probability as specified by the ProteinLynx Global Server 2.2. Protein identifications were accepted if they could be established at greater than $95 \%$ probability and contained at least 1 identified peptide. When several accession numbers in the database matched the same set of peptides identified, the entries with the most descriptive name were reported. For all identifications based on one peptide sequence, the MS/MS spectrum was sequenced and reinterpreted manually using Mass-Lynx 4.0 Protein Sequencing modules (PepSeq, Waters).

\section{Western Blotting}

For immunoblotting, $20 \mu \mathrm{g}$ protein samples were separated by SDS-PAGE (4-20\% polyacrylamide gradient gel) and transferred on nitrocellulose (Millipore, Bedford, MA). The membrane was developed in accordance with a protocol provided by LI-COR, Inc. Biosciences. Bit1 protein was identified using anti-Bit1 primary antibody. The reactive bands were revealed and detected with the Odyssey ${ }^{\mathrm{TM}}$ Infrared Imaging System, as previously reported [21,22].

\section{RESULTS AND DISCUSSION}

\section{Cells Deficient in p21 are Hypersensitive to Topotecan}

To determine the impact of p21 on the sensitivity of tumor cells to topotecan, wild type and p21-/- HCT-116 cells were exposed for 24 hours to various doses of the drug. Cellular viability was determined by the crystal violet assay. As can be seen from the data in Fig. (1), p21-/- cells were much more sensitive to topotecan treatment than wild-type cells. The inhibitory concentration $\left(\mathrm{IC}_{50}\right)$ of topotecan for HCT-116 cells was found to be $11.8 \mu \mathrm{M}$, compared to only $1.15 \mu \mathrm{M}$ for $\mathrm{p} 21-/-$ cells. The disparity in antiproliferative responses of HCT-116 isogenic cells probably reflects the higher sensitivity of p21-/- cells to topotecan-induced cell death [24,25].

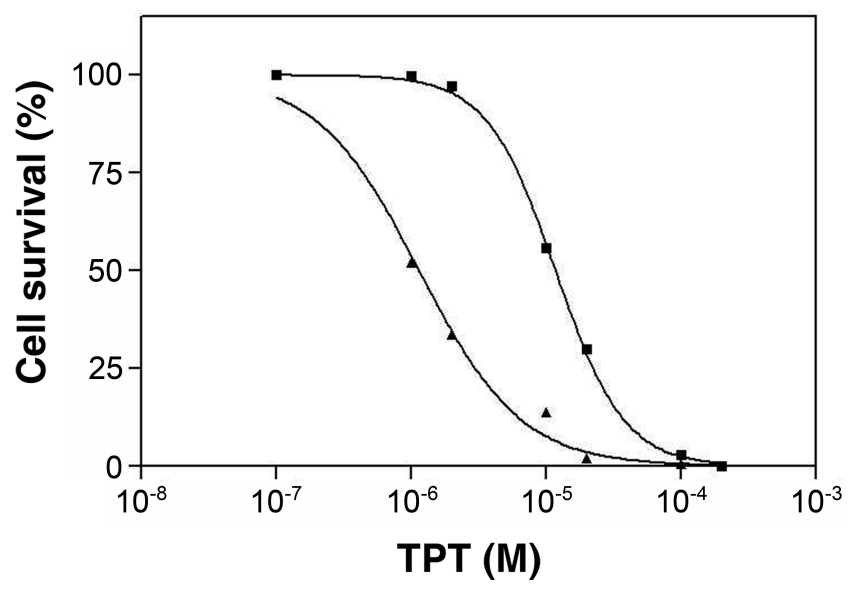

Fig. (1). Topotecan sensitivity in human colon carcinoma cell lines. Dose-response curves of HCT-116 (ם), and HCT-116 $(\mathrm{p} 21-/-)(\boldsymbol{\Delta})$ cells following treatment with various concentrations of topotecan (TPT) for $24 \mathrm{~h}$, as determined by the crystal violet assay. Data are mean values of two independent experiments, where each concentration of drug was assayed in four wells of a 48-well plate, and are expressed as mean percentages of optical density values compared with vehicle (DMSO) treated cells.

\section{Multidimensional Separation of Subcellular Fractions by SDS-PAGE}

Cell cultures were treated for 24 hours with sub-toxic doses $(1 \mu \mathrm{M})$ of topotecan. The use of this dose allows assessing the role of p21 in topotecan-induced stress responses without the complications of excessive cell death. Enriched nuclei, mitochondrial, and cytosolic fractions of the isogenic cells were then separated by one-dimensional electrophoresis (SDS-PAGE) prior to protein identification by mass spectrometry. While primarily used to fractionate simple protein mixtures to homogeneity prior to identification of individual species by peptide mass mapping, SDS-PAGE has been used to identify large numbers of protein components in biological complexes [26,27]. As summarized in Fig. (2), the entire gel lane was systematically cut into equal size pieces of $1 \mathrm{~cm}$ (18 slices) and each gel slice was digested separately with trypsin, as previously reported $[21,22]$. The extracted peptides from the gel slices were analyzed using LC-ESIMS/MS to identify the proteins. The use of SDS-PAGE in the first dimension denatures the proteins and transforms one very complex mixture into several less complex mixtures. In addition, the technique is very effective at removing excess reagents such as salts from the original protein mixture prior to in-gel digestion. The molecular weight ranges of identified proteins in each gel slice were used to increase the confidence of the identification. The predicted molecular weights of the identified proteins correlated well with the size range of the gel slices.

\section{Subcellular Protein Identification}

Digested proteins in each gel slice were identified using MS/MS mass spectra analysis and peptide sequencing, as described in Materials and Methods. The complete list of proteins identified is summarized in Supplemental data Table 1; this table lists the number of peptides observed for each identified protein, the probability $\%$ of identification, and the accession number of each protein for each cell line 


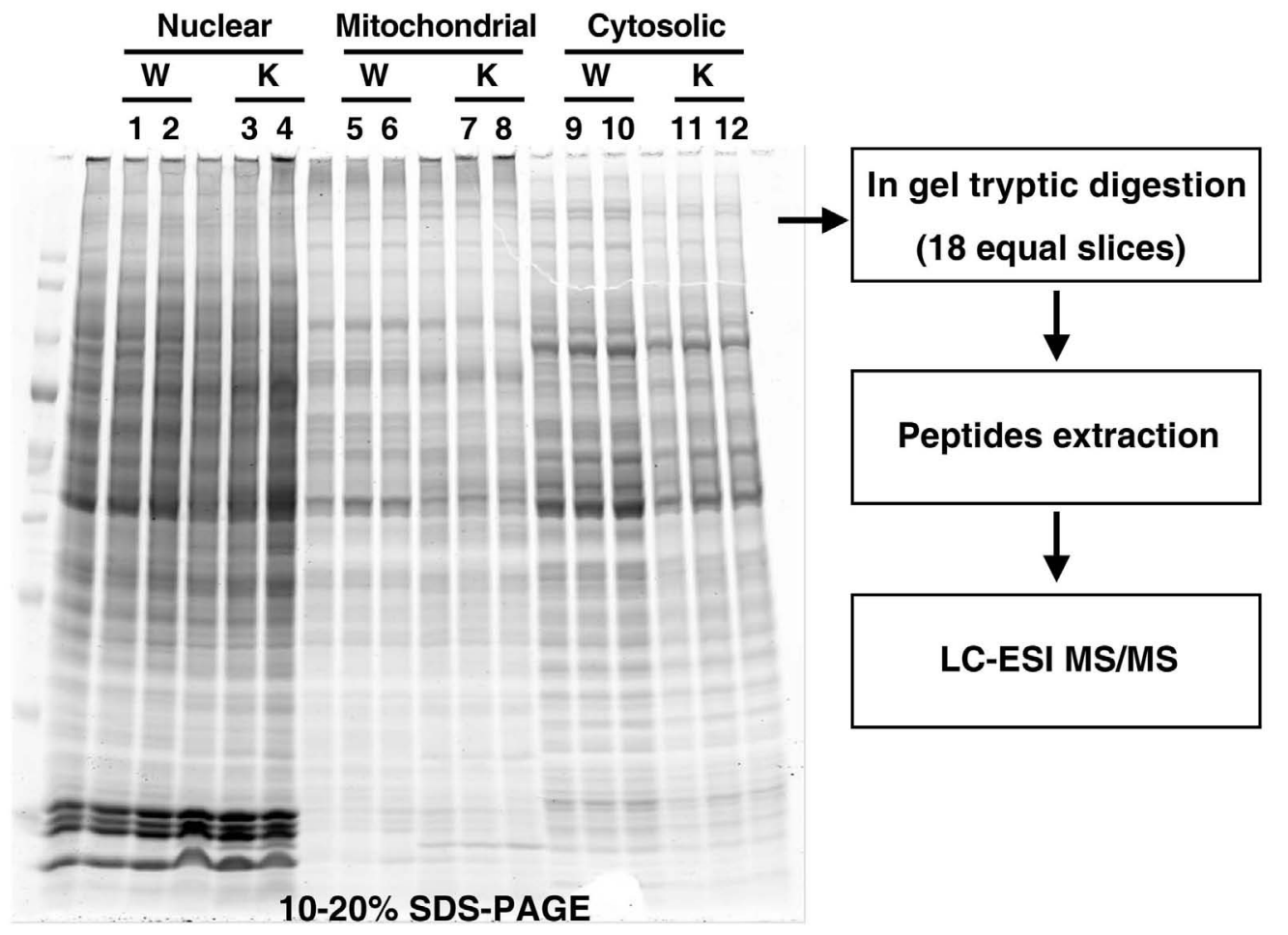

Fig. (2). Approach used for subcellular proteome analysis of topotecan-treated colon carcinoma cells. Subcellular fractions of HCT-116 and HCT-116 (p21-/-) cells were separated by one-dimensional electrophoresis prior to protein identification by mass spectrometry. W= HCT-116 wild type cells; K= p21 knockout HCT-116 cells. Odd numbers = cells treated with vehicle $(\mathrm{DMSO})$; even numbers = cells treated with topotecan (TPT).

after treatment with either DMSO, or TPT. Totally, 174 unique proteins were deregulated in HCT-116 following treatment with TPT, whereas only 146 proteins were identified in HCT-116 p21-/- cells. As can be seen from the data presented in Table 1, 79 (32.2\%), 46 (28.9\%), and 33 (20.9\%) uniquely up-regulated proteins were identified, respectively, in nuclear, mitochondrial and cytosolic fractions of HCT-116 cells after TPT treatment; whereas 45 (26.2\%), $29(35.8 \%)$, and $25(24.8 \%)$ overexpressed proteins were identified, respectively, in nuclear, mitochondrial, and cytosolic fractions of HCT-116 p21-/- cells following drug treatment. Of the total 174 deregulated proteins identified in HCT-116 cells, 74 proteins $(21.1 \%)$ were expressed from only control-treated cells and 100 proteins $(26.6 \%)$ from TPT-treated cells. The results indicate that 74 proteins were down regulated in response to TPT treatment, whereas 100 proteins were upregulated in HCT-116 cells. In HCT-116 p21-/- cells, 89 proteins $(33.1 \%)$ were down regulated and 57 proteins $(22.4 \%)$ were up regulated after TPT treatment. Thus, the functionality of p 21 has a significant effect on the number of identified proteins. For example, in our study we have identified the expression of $\beta$-2-microglobulin protein (accession \# P61769, Supplemental data Table 2-2) in HCT116 cells after TPT treatment, which is down regulated in p21-/- cells. $\beta$-2-microglobulin has been found to induce apoptosis in leukemia cell lines [28] and suppresses the growth of isolated myeloma tumors by inducing apoptosis and cell cycle arrest [29]. Recently, $\beta$-2-microglobulin was also found to be induced by p21-overexpressing tumor cells [30]. Similarly, we identified the expression of galectin-12 protein in HCT-116 cells (accession \# Q96DT0, Supplemental data Table 2-2) following TPT treatment, but down-

Table 1. Numbers of Deregulated Proteins Identified in Both HCT-116 \& HCT-116 p21-/- Cells After DMSO and Topotecan (TPT) Treatment ${ }^{\mathrm{a}}$

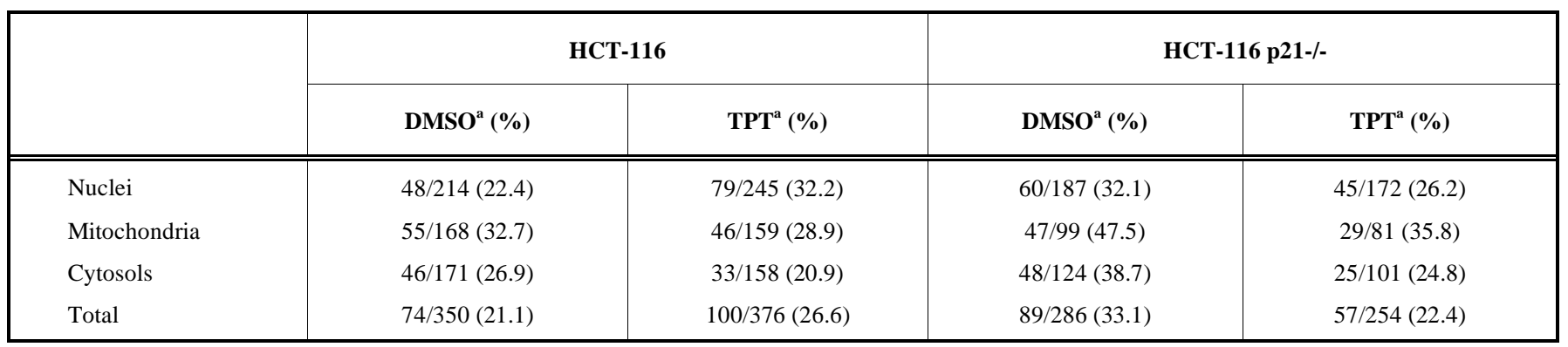

${ }^{\mathrm{a}}$ Deregulated proteins/total subcellular proteins. 
regulated in p21-/- cells. Galectins constitute a family of proteins that bind to $\beta$-galactoside residues and have diverse physiological functions. For example, galectin-12 has been shown to be involved in the regulation of apoptosis through interactions with many intracellular proteins [31,32], and its expression is induced by p21 expression [33]. Another protein that was identified to be over expressed in HCT-116 cells following treatment with TPT, but not in p21-/- cells is Lamin B1 (accession \# P20700, Supplemental data Table 22). Lamin B1 has been found to be associated with the processes of necrosis and apoptosis following the application of apoptotic stimulus [34,35]. This cell death-related effect is indirectly regulated by $\mathrm{p} 21$ overexpression in cells [33]. Collectively, the data indicate that the functionality of $\mathrm{p} 21$ is an important factor for the activation of stress-responsive proteins in cells. It is worthy to note that the functionality of p53 has also been shown to have a significant effect on stressresponse induced genes. Using cDNA microarray technology, we previously showed that $10 \%$ of transcripts were deregulated in response to TPT treatment in HCT-116 cells, whereas only $1 \%$ of the transcripts changed in HCT-116 p53-/- cells [23]. Since HCT-116 and HCT-116 p21-/- cells both express a wild type p53, we conclude that the change seen in protein expression as a result of topotecan-induced stress response is associated with the functionality of p21 in cells.

For data acquisition and protein identification, Supplemental data Fig. (1) illustrates an example of MS/MS representative spectra of proteins identified on the basis of a single peptide. The sequence TAPEALSYR was matched to amino acids 784-791 of Myosin-10 (Supplemental data Fig. (1a), accession \# P35580, Supplemental data Table 2-1), and the sequence TPAQFDADELR to amino acids 772-782 of Ephrin type-A receptor 2 (Supplemental data Fig. (1b), accession \# P29317, Supplemental data Table 2-4). The functional distribution of all identified proteins is shown in Figs. (3 and 4). They were classified into cell cycle, apoptosis and oxidative stress, cell energy, cell metabolism, cell structure and motility, cytoskeleton and membrane trafficking, nucleic acid metabolism, protein metabolism and modification, and signal transduction. As can be seen from the functional distribution data in Fig (4A), the total percentage of identified proteins involved in nucleic acid metabolism, cell transport and trafficking are repressed in the nuclear fractions of $\mathrm{p} 21-$ /- cells after TPT treatment, as compared to HCT-116 cells (Fig. 3A). As topoisomerase I inhibitors (TOPI), camptothecin analogues such as TPT are known to target DNA by generating replication-mediated double-strand breaks and inhibiting DNA religation [20,23]. Thus the expression of many proteins involved in the cellular process of transcription, translation, and degradation of proteins are usually deregulated by TOPI [36]. In this study, several other proteins known to be involved in mRNA processing like DNA (cytosine-5)-methyltransferase 1, Matrin 3, DNA replication licensing factor MCM4, heterogeneous nuclear ribonucleoprotein $\mathrm{Q}$, poly(Rc)-binding protein 1, and splicing factor arginine serine rich 7 (Supplemental data Table 2) were also found to be repressed in p21-/- cells. All these alterations in protein expression could significantly affect major biological processes of cells like transcription, mRNA processing and translation. Hence the data provided the first clues about the hypersensitivity of HCT-116 p21-/- cells to TPT treatment seen in Fig. (1). The hypersensitivity to TPT could partly be related to the stress responses of p21-/- cells as a result of the down regulation of protein-associated with nucleic acid metabolism, which could lead to the shut down of its transcription machinery.

It has been known that cell death is a complex biochemical process involving a cascade of closely coordinated factors in different cellular compartments such nuclei, mitochondrial, and cytosols which converge in the activation of intracellular factors and caspases and their modification of protein substrates within the nucleus and cytoplasm $[37,38]$. In the mitochondrial fractions of cells, we were able to identify many proteins that are associated with the mitochondrial functions. As can be seen from the functional distribution data of deregulated proteins in Fig. (4B), the number of proteins associated with cell signaling and protein metabolism and modification in p21-/- cells are higher than that of HCT116 cells (Fig. 3B) following TPT treatment. In the mitochondrial fractions of HCT-116 p21-/- cells, we were able to identify proteins that are involved in protein modifications such as ubiquitin (UBB), translocon-associated protein subunit delta (SSR4), protein FAM62A, caveolin 1 (CAV1) and ATP synthase O subunit (ATP50). These proteins are repressed in the mitochondrial fractions of HCT-116 cells treated with TPT (Supplemental data Table 2-10). Of interest to note is the identification of peptidyl-tRNA hydrolase2 (Bit1) protein in p21-/- cells (accession\# Q9YE5, Supplemental data Table 2-10). The Bcl-2 inhibitor of transcription (Bit1) is a mitochondrial protein that functions as a peptidyltRNA hydrolase, but when released into the cytoplasm, it elicits caspase-independent apoptosis [39], possibly by forming a complex with the transcriptional co-regulator, AES (amino-terminal enhancer of split) [40]. It has been also reported that the proapoptotic function of Bit1 is uniquely counteracted by integrin-mediated cell attachment [41]. As can be seen from the western blot in Fig. (5), the level of the upper band of Bit1 protein in HCT-116 p21-/- cells treated with TPT for $24 \mathrm{~h}$ is higher than its level in the control or TPT treated HCT-116 cells. We conclude that Bit1 is expressed in both cell lines but its detection level in HCT-116 cells is very low. This indicates that the proapoptotic effect of the released of Bit1 protein could be one of the molecular mechanisms by which HCT-116 p21-/- cells is accelerated to be more responsive to TPT-induced cell death. In contrast, the identification of Integrin $\beta 4$ protein (accession\# P16144, Supplemental data Table $\mathbf{2 - 4}$ ) in HCT-116 cells following TPT treatment could counteract this pro-apoptotic effect, and hence these cells are less responsive to TPT treatment than p21-/- cells [39]. It would be of great interest to study further the potential role of Bit1 and p21-mediated signaling pathways.

Among the cytosolic proteins that were identified to be upregulated in HCT-116 p21-/- cells after TPT treatment, but repressed in HCT-116 cells are: glucose-6-phosphate dehydrogenase (G-6PD) (accession \# P11413, Supplemental data Table 2-12) and glutathione S-transferase P (GSTP1) (accession \# P09211, Supplemental data Table 2-12). Both proteins are involved in the cellular energy metabolism and protect cells against oxidative stress by maintaining optimal cellular detoxifying machinery $[42,43]$. The identification of these cytosolic proteins in the absence of functional p21 indicates that their release is a result of a stress response following 

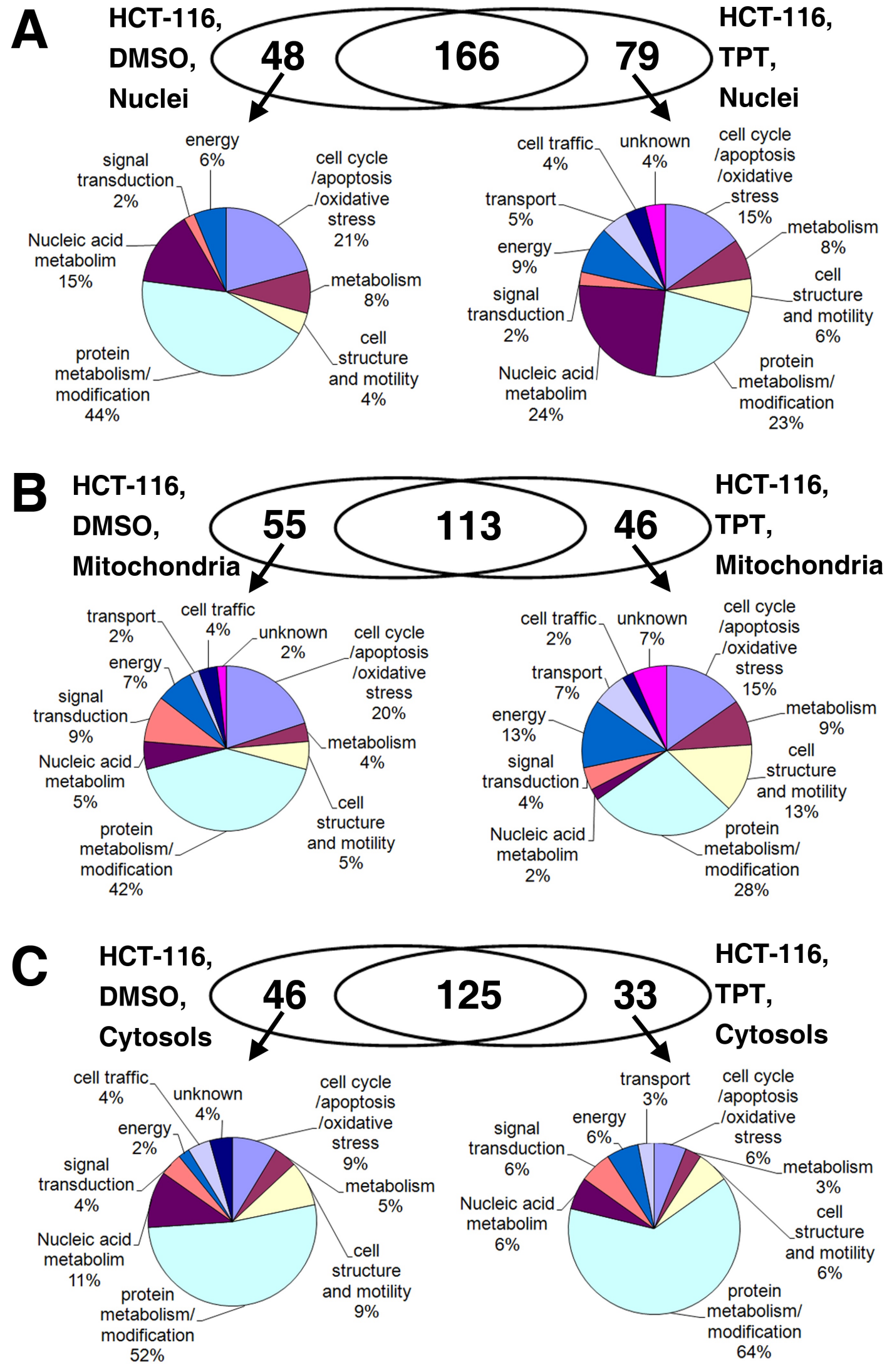

Fig. (3). Functional distribution of subcellular proteins isolated from 1D-PAGE gels and identified by MS in HCT-116 cells after treatment with DMSO or TPT. A: Functional proteins identified in nuclear fractions; B: Functional proteins identified in mitochondrial fractions; C: Functional proteins identified in cytosolic fractions. 

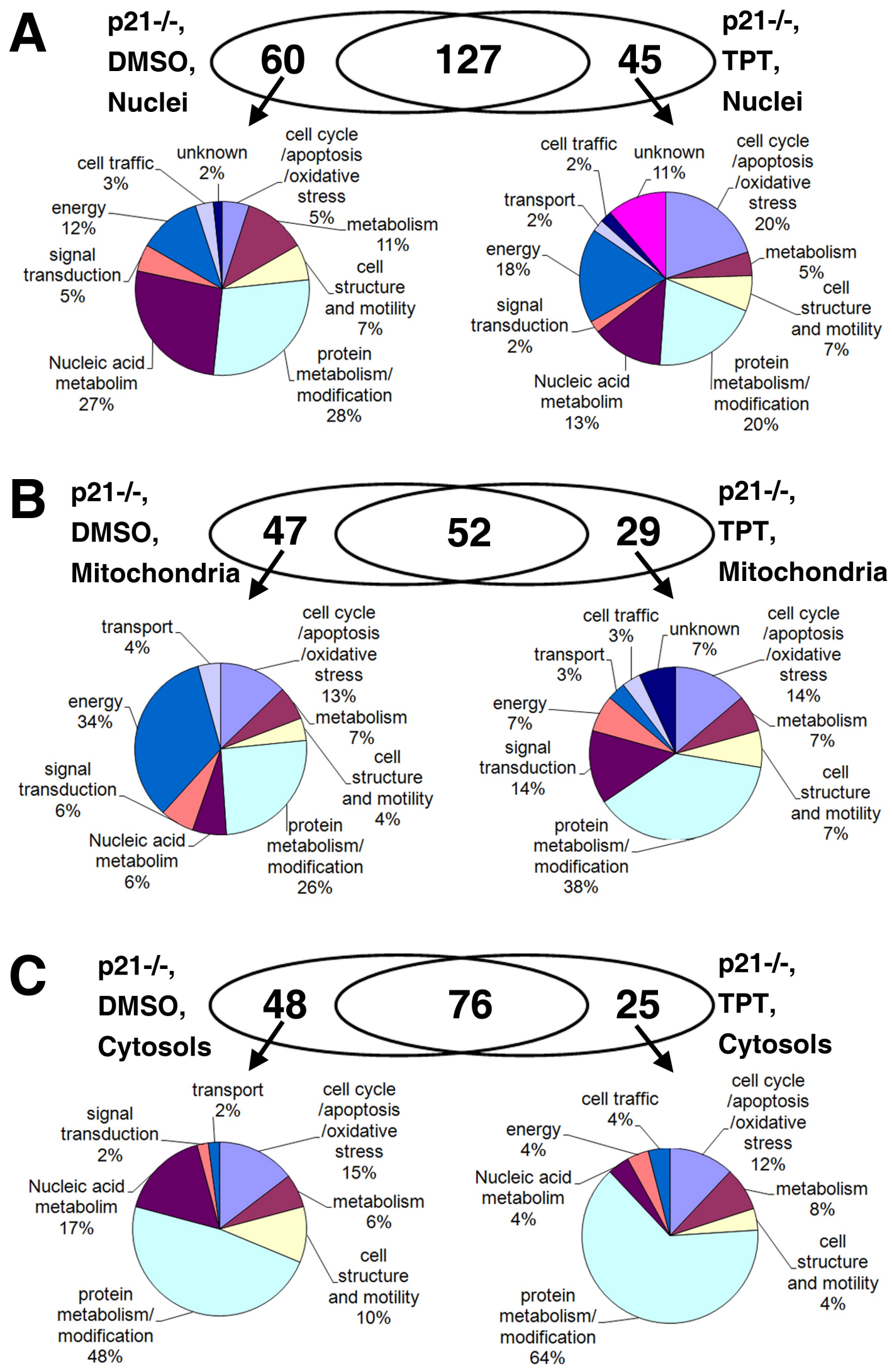

Fig. (4). Functional distribution of subcellular proteins isolated from 1D-PAGE gels and identified by MS in HCT-116 (p21-/-) cells after treatment with DMSO or TPT. A: Functional proteins identified in nuclear fractions; B: Functional proteins identified in mitochondrial fractions; C: Functional proteins identified in cytosolic fractions. 


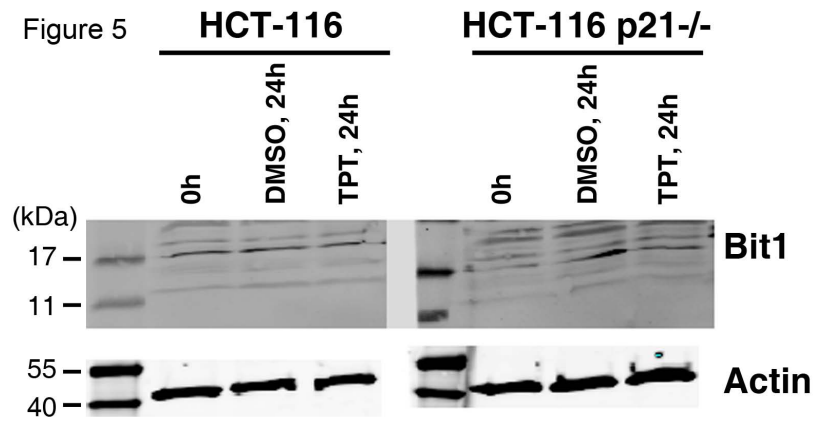

Fig. (5). Western blot of Bit1 protein from control and TPTtreated cells. Cells were treated with $1 \mu \mathrm{M}$ TPT or vehicle control for $24 \mathrm{~h}, 20 \mu \mathrm{g}$ of protein samples of cell lysates from the whole cell extracts were separated by SDS-PAGE (4 to $20 \%$ polyacrylamide) and Western blotted with antibody against Bit1 (the Bit1 protein typically appears as a double band). $\beta$-Actin was used as a loading control. The reactive bands were detected with the Odyssey $^{\mathrm{TM}}$ Infrared Imaging System.

treatment with DNA damaging agents such as topotecan [44]. In addition, we are able to identify proteins involved cell trafficking such as ADP ribosylation factor 4 (ARF4) following TPT treatment of p21-/- cells but not HCT-116 cells (accession \# P18085, Supplemental data file Table 212). ARF4 is a GTP-binding protein that function is an ADPribosyltransferase and involved in protein trafficking from Golgi apparatus into the endoplasmic reticulum (ER). It has been observed that the activation of the protein trafficking mechanism is triggered by the up-regulation of ARF4, and is a characteristic of cells distant to undergo oxidative stressinduced cell death [45,46]. As a result of this oxidative stress, more proteins that are involved in cell trafficking, and cellular energy metabolism were identified in p21-/- cells following treatment with TPT (Fig. 4C), as compared to HCT-116 cells (Fig. 3C). Furthermore, the identification of the macrophage immigration inhibitory factor (MIF) protein in the cytosolic fractions of p21-/- cells following TPT treatment (accession \# P14174, Supplemental data Table 212) provided an additional support that caspase-independent pathways such as the tumor necrosis factor $\alpha$ (TNF $\alpha$ )mediated response could also be involved in the hypersensitivity of p21-/- cells to DNA damage response (Fig. 1). MIF is a ubiquitously expressed proinflammatory mediator that has also been implicated in oncogenic transformation and tumor progression [47]. Recent insights into the pleiotropic activities of endogenous MIF showed that it also plays an essential role in cell cycle arrest and in the DNA damage response of cells [48,49]. MIF has also been shown to be involved in TNF $\alpha$-mediated cell death. For example, apoptotic mature neutrophils were shown to release MIF upon stimulation with TNF $\alpha$ [50], and that retinoic acid-mediated apoptosis has been shown to involve the expression of apoptosis related genes like MIF, IGIF and TNF $\alpha$ [51]. In order for $\mathrm{TNF} \alpha$-mediated cell death to occur, $\mathrm{TNF} \alpha$ has to bind to two different cell surface receptors, the TNF receptor type I (TNFRI) and the TNF receptor type II (TNFRII) [52]. The binding of TNF $\alpha$ - to these receptors recruits cytosolic proteins such as TRADD, TRAP2 and TRAF2 to initiate signaling to downstream effectors [53]. It is of interest to note, that we have also identified the expression of cytosolic protein TRAP2 in p21-/- cells following TPT treatment (accession \# Q13200, Supplemental data Table 2-12), but not in HCT-116 cells. These observations clearly indicate that $\mathrm{TNF} \alpha-$ mediated cell death is part of the molecular mechanisms by which p21-/- cells is more responsive to TPT treatment as compared to HCT-116 cells (Fig. 1). Furthermore, these observations indicate that lack of a functional p21 protein accelerates caspase-independent cell death induced by TPT in colon cancer cells.

\section{CONCLUSION}

An increased knowledge in the changes of protein profiles in response to pharmacological stress is important in understanding the role of $\mathrm{p} 21$ in DNA damage response. This study used a gel-based mass spectrometry proteomic approach to identify differentially deregulated proteins in the subcellular fractions of isogenic p21 null (p21-/-) and wildtype human HCT-116 cells following treatment with sublethal doses of topotecan (TPT). The detailed analysis of the identified proteins presented, for the first time, the basis for possible molecular mechanisms of TPT-induced cell death in p21 knockdown cells. In this context, most of the identified proteins had not been reported to be associated with p21 signaling. Further investigations on the role these deregulated proteins would shed new insights into developing new therapeutic approaches for targeting p21 signaling pathways.

\section{ACKNOWLEDGEMENTS}

This work is supported in part by grant no. BCTR0402398 to SSD from the Susan G. Komen Foundation for Breast Cancer Research. HCT-116 and an isogenic p21 knockout cell line were kindly provided by Dr. Bert Vogelstein (John Hopkins Medical Institute, Baltimore, MD).

\section{ABBREVIATIONS}

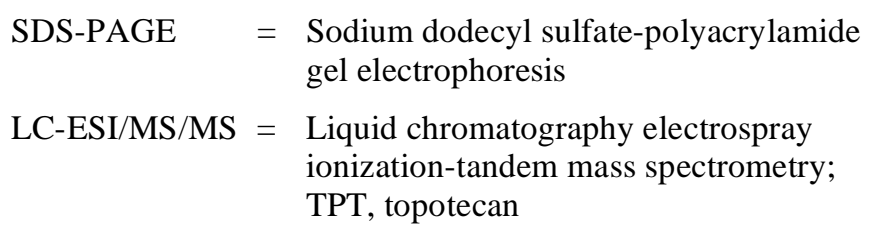

\section{SUPPLEMENTAL DATA}

Supplementary material can be viewed at: http://www. bentham.org/open/toprotj.

Table 1. A complete list of identified proteins using gelbased mass spectrometry approach.

Table 2. A list of deregulated proteins in the subcellular fractions of isogenic p21-null (p21-/-) and wild-type human HCT-116 colon carcinoma cells following DMSO or topotecan (TPT) treatment.

Fig. (1). MS/MS representative spectra of proteins identified on the basis of a single peptide. The sequence TAPEALSYR was matched to amino acids 784-791 of Myosin-10 (A) and the sequence TPAQFDADELR to amino acids 772-782 of Ephrin type-A receptor 2 (B). Ions from "y" series are shown. 


\section{REFERENCES}

[1] Kao, J.; Rosenstein, B.S.; Peters, S.; Milano, M.T.; Kron, S.J. Cellular response to DNA damage. Ann. N. Y. Acad. Sci., 2005, 1066, 243-58.

[2] Zhou, J.; Lim, C.U.; Li, J.J.; Cai, L.; Zhang, Y. The role of NBS1 in the modulation of PIKK family proteins ATM and ATR in cellular response to DNA damage. Cancer Lett., 2006, 243, 9-15.

[3] Hurley, P.J.; Bunz, F. ATM and ATR: components of an integrated circuit. Cell Cycle, 2007, 6, 414-7.

[4] Vousden, K.H.; Prives, C. p53 and prognosis: new insights and further complexity. Cell, 2005, 120, 7-10.

[5] Strano, S.; Dell'Orso, S.; Di Agostino, S.; Fontemaggi, G.; Sacchi, A.; Blandino, G. Mutant p53: an oncogenic transcription factor. Oncogene, 2007, 26, 2212-9.

[6] Brooks, C.L.; Gu, W. p53 ubiquitination: Mdm2 and beyond. Mol. Cell, 2006, 21, 307-15.

[7] Pereg, Y.; Shkedy, D.; de Graaf, P.; Meulmeester, E.; EdelsonAverbukh, M.; Salek, M.; Biton, S.; Teunisse, A.F.; Lehmann, W.D.; Jochemsen, A.G.; Shiloh, Y. Phosphorylation of Hdmx mediates its Hdm2- and ATM-dependent degradation in response to DNA damage. Proc. Natl. Acad. Sci. USA, 2005, 102, 5056-61.

[8] Bartek, J.; Lukas, J. Pathways governing G1/S transition and their response to DNA damage. FEEBS Lett., 2001, 490, 117-122.

[9] Bae, K.M.; Wang, H.; Jiang, G.; Chen, M.G.; Lu, L.; Xiao, L. Protein kinase $\mathrm{C}$ epsilon is overexpressed in primary human nonsmall cell lung cancers and functionally required for proliferation of non-small cell lung cancer cells in a p21/Cip1-dependent manner. Cancer Res., 2007, 67, 6053-63.

[10] Ciccarelli, C.; Marampon, F.; Scoglio, A.; Mauro, A.; Giacinti, C.; De Cesaris, P.; Zani, B.M. p21 WAF1 expression induced by MEK/ERK pathway activation or inhibition correlates with growth arrest, myogenic differentiation and onco-phenotype reversal in rhabdomyosarcoma cells. Mol. Cancer, 2005, 4, 41-59.

[11] Zheng, Q.H.; Ma, L.W.; Zhu, W.G.; Zhang, Z.Y.; Tong, T.J. p21Waf1/Cip1 plays a critical role in modulating senescence through changes of DNA methylation. J. Cell Biochem., 2006, 98, 1230-48.

[12] Lee, S.; Helfman, D.M. Cytoplasmic p21Cip1 is involved in Rasinduced inhibition of the ROCK/LIMK/cofilin pathway. J. Biol. Chem., 2004, 279, 1885-91.

[13] Itoh, T.; Linn, S. The fate of p21CDKN1A in cells surviving UVirradiation. DNA Repair (Amst.), 2005, 4, 1457-62.

[14] Deng, C.; Zhang, P.; Harper, J.W; Elledge, S.J.; Leder, P. Mice lacking p21CIP1/WAF1 undergo normal development, but are defective in G1 checkpoint control. Cell, 1995, 82, 675-84.

[15] Gorospe, M.; Cirielli, C.; Wang, X.; Seth, P.; Capogrossi, M.C.; Holbrook, N.J. p21 (Waf1/Cip1) protects against p53-mediated apoptosis of human melanoma cells. Oncogene, 1997, 14, 929-935.

[16] Li, W.W.; Fan, J.; Hochhauser, D.; Bertino, J.R. Overexpression of p21 waf1 leads to increased inhibition of E2F-1 phosphorylation and sensitivity to anticancer drugs in retinoblastoma-negative human sarcoma cells. Cancer Res., 1997, 57, 2193-99.

[17] Fan, S.; Chang, J.K.; Smith, M.L.; Duba, D.; Fornace, A.J.; O'Connor, P.M. Cells lacking CIP1/WAF1 genes exhibit preferential sensitivity to cisplatin and nitrogen mustard. Oncogene, 1997, 14, 2127-36.

[18] Bunz, F.; Hwang, P.M.; Torrance, C.; Waldman, T.; Zhang, Y.; Dillehay, L.; Williams, J.; Lengauer, C.; Kinzler, K.W.; Vogelstein, B. Disruption of p53 in human cancer cells alters the responses to therapeutic agents. J. Clin. Invest., 1999, 104, 263-269.

[19] Kollmannsberger, C.; Mross, K.; Jakob, A.; Kanz, L.; Bokemeyer, C. Topotecan, a novel topoisomerase I inhibitor: pharmacology and clinical experience. Oncology (Basel), 1999, 56, 1-12.

[20] Pommier Y. Topoisomerase I inhibitors: camptothecins and beyond. Nat. Rev. Cancer, 2006, 6, 789-802.

[21] Rehman, A.; Chahal, M.S.; Tang, X.; Bruce, J.E.; Pommier, Y.; Daoud, S.S. Proteomic identification of heat shock protein 90 as a candidate target for p53 mutation reactivation by PRIMA-1 in breast cancer cells. Breast Cancer Res., 2005, 7, R765-74.

[22] Lee, K.; Wang, T.; Paszczynski, A.J.; Daoud, S.S. Expression proteomics to p53 mutation reactivation with PRIMA-1 in breast cancer cells. Biochem. Biophys. Res. Commun., 2006, 349, 111724.

[23] Daoud, S.S.; Munson, P.J.; Reinhold, W.; Young, L.; Prabhu, V.V.; Yu, Q.; LaRose, J.; Kohn, K.W.; Weinstein, J.N.; Pommier, Y. Impact of p53 knockout and topotecan treatment on gene expression profiles in human colon carcinoma cells. A pharmacogenomic study. Cancer Res., 2003, 63, 2782-93.

[24] Chan, T.A.; Hwang, P.M.; Hermeking, H.; Kinzler, K.W.; Vogelstein, B. Cooperative effects of genes controlling the G2/M checkpoint. Genes Dev., 2000, 14, 1584-1588.

[25] Efeyan, A.; Collado, M.; Velasco-Miguel, S.; Serrano, M. Genetic dissection of the role of p21Cip1/Waf1 in p53-mediated tumor suppression. Oncogene, 2007, 26, 1645-1649.

[26] Rappsilber, J.; Ryder, U.; Ryder, U.; Lamond, A.I.; Mann, M. Large-scale proteomic analysis of the human spliceosome. Genome Res., 2002, 12, 1231-45.

[27] de Souza, G.A.; Godoy, L.M. F.; Mann, M. Identification of 491 proteins in the tear fluid proteome reveals a large number of proteases and protease inhibitors. Genome Biol., 2006, 7, R72-R83.

[28] Gordon, J.; Wu, C.H.; Rastegar, M.; Safa, A.R. Beta-2microglobulin induces caspase-dependent apoptosis in the CCRFHSB-2 human leukemia cell line independently of the caspase-3, -8 and -9 pathways but through increased reactive oxygen species. Int. J. Cancer, 2003, 103, 316-327.

[29] Min, R.; Li, Z.; Epstein, J.; Barlogie, B.; Yi, Q. Beta-2microglobulin as a negative growth regulator of myeloma cells. $\mathrm{Br}$. J. Haematol., 2002, 118, 495-505.

[30] Currid, C.A.; O’Connor, D.P.; Chang, B-D.; Gebus, C.; Harris, N.; Dawson, K.A.; Dunn, M.J.; Pennington, S.R.; Roninson, I.B.; Gallagher, W.M. Proteomic analysis of factors released from p21overexpressing tumor cells. Proteomics, 2006, 6, 3739-3753.

[31] Hsu, D.K.; Yang, R.Y.; Liu, F.T. Galectins in apoptosis. Methods Enzymol., 2006, 417, 256-73.

[32] Beer, A.; André, S.; Kaltner, H.; Lensch, M., Franz, S.; Sarter, K.; Schulze, C; Gaipl, U.S.; Kern, P.; Herrmann, M.; Gabius, H.J. Human galectins as sensors for apoptosis/necrosis-associated surface of granulocytes and lymphocytes. Cytometry A., 2008, 73, 139-47.

[33] Roninson, I.B. Oncogenic functions of tumor suppressor p2 $1^{\text {Waf1/Cip1/Sdi1 }}$ : association with cell senescence and tumorpromoting activities of stromal fibroblasts. Cancer Lett., 2002, 179, $1-14$.

[34] Broers, J.L.; Bronnenberg, N.M.; Kuijpers, H.J.; Schutte, B.; Hutchinson, C.J.; Ramaekers, F.C. Partial cleavage of A-type lamins occurs with their total disintegration from the nuclear lamina during apoptosis. Eur. J. Cell. Biol., 2002, 81, 677-91.

[35] Sato, A.; Hiramoto, A.; Satake, A.; Miyazaki, E.; Naito, T.; Wataya, Y.; Kim, H.S. Association of nuclear membrane protein lamin b1 with necrosis and apoptosis in cell death induced by 5-fluoro-2'deoxyuridine. Nucleosides Nucleotides Nucleic Acids, 2008, 27, 433-8.

[36] Yu, Y.; Wang, L.S.; Shen, S.M.; Xia, L.; Zhang, L.; Zhu, Y.S.; Chen, G.Q. Subcellular proteome analysis of camptothecin analogue NSC606985-treated acute myeloid leukemia cells. $J$. Proteome Res., 2007, 6, 3808-18.

[37] Hansen, J.M.; Go, Y.M.; Jones, D.P. Nuclear and mitochondrial compartmentation of oxidative stress and redox signaling. Annu. Rev. Pharmacol. Toxicol., 2006, 46, 215-34.

[38] Kroemer, G.; Galluzzi, L.; Brenner C. Mitochondrial membrane permeabilization in cell death. Physiol. Rev., 2007, 87, 99-163.

[39] Kairouz-Wahbe, R.; Biliran, H.; Luo, X.; Khor, I.; Wankell, M.; Besch-Williford, C.; Pascual, C; Oshima, R.; Ruoslahti, E. Anoikis effector Bit1 negatively regulates Erk activity. Proc. Natl. Acad. Sci. USA, 2008, 105, 1528-32.

[40] Jan, Y.; Matter, M.; Pai, J.T.; Chen, Y.L.; Pilch, J.; Komatsu, M.; Ong, E.; Fukuda, M.; Ruoslahti, E. A mitochondrial protein, Bit1, mediates apoptosis regulated by integrins and Groucho/TLE corepressors. Cell, 2004, 116, 751-62.

[41] Stupack, D.G.; Cheresh, D.A. A Bit-role for integrins in apoptosis. Nat. Cell Biol., 2004, 6, 388-389.

[42] Préville, X.; Salvemini, F.; Giraud, S.; Chaufour, S.; Paul, C.; Stepien, G.; Ursini, M.V.; Arrigo, A.P. Mammalian small stress proteins protect against oxidative stress through their ability to increase glucose-6-phosphate dehydrogenase activity and by maintaining optimal cellular detoxifying machinery. Exp. Cell Res., 1999, 247, 61-78.

[43] Fico, A.; Paglialunga, F.; Cigliano, L.; Abrescia, P.; Verde, P.; Martini, G.; Iaccarino, I.; Filosa, S. Glucose-6-phosphate dehydrogenase plays a crucial role in protection from redox-stress-induced apoptosis. Cell Death Differ., 2004, 11, 823-31.

[44] Bravard, A.; Petridis, F.; Luccioni, C. Modulation of antioxidant enzymes p21WAF1 and p53 expression during proliferation and 
differentiation of human melanoma cell lines. Free Radic. Biol. Med., 1999, 26, 1027-33.

[45] Suzl, T.; Grabaski, R.; Lyons, S.; Morohashi, Y.; Shestopal, S.; Lowe, M.; Sztul, E. Dissecting the role of the ARF guanine nucleotide exchange factor GBF1 in Golgi biogenesis and protein trafficking. J. Cell Sci., 2007, 120, 3929-40.

[46] Colussi, C.; Albertini, M.C.; Coppola, S.; Rovidati, S.; Galli, F.; Ghibelli, L. H2O2-induced block of glycolysis as an active ADPribosylation reaction protecting cells from apoptosis. FASEB $J$., 2000, 14, 2266-76.

[47] Mitchell, R.A. Mechanisms and effectors of MIF-dependent promotion of tumourigenesis. Cell. Signal., 2004, 16, 13-19.

[48] Fingerle-Rowson, G.; Petrenko, O. MIF coordinates the cell cycle with DNA damage checkpoints. Lessons from knockout mouse models. Cell Div., 2007, 2, 22-29.

[49] Nemajerova, A.; Moll, U.M.; Petrenko, O.; Fingerle-Rowson, G. Macrophage migration inhibitory factor coordinates DNA damage response with the proteasomal control of the cell cycle. Cell Cycle, 2007, 6, 1030-4.
[50] Daryadel, A.; Grifone, R.F.; Simon, H.U.; Yousefi, S. Apoptotic neutrophils release macrophage migration inhibitory factor upon stimulation with tumor necrosis factor-alpha. J. Biol. Chem., 2006, 281, 27653-61.

[51] Sarkar, S.A.; Sharma, R.P. Expression of selected apoptosis related genes, MIF, IGIF, and TNF alpha during retinoic-acid-induced neural differentiation in murine embryonic stem cells. Cell Struct.. Funct., 2002, 27, 99-107.

[52] Wallach, D.; Varfolomeev, E.E.; Malinin, N.L.; Goltsev, Y.V.; Kovalenko, A.V.; Boldin, M.P. Tumor necrosis factor receptor and Fas signaling mechanisms. Annu. Rev. Immunol., 1999, 17, 331-67.

[53] Kuai, J.; Wooters, J.; Hall, J.P.; Rao, V.R.; Nickbarg, E.; Li, B.; Chatterjee-Kishore, M.; Qiu, Y.; Lin, L.L. NAK is recruited to the TNFR1 complex in a TNFo-dependent manner and mediates the production of RANTES. Identification of endogenous TNFRinteracting proteins by a proteomic approach. J. Biol. Chem., 2004, $279,53266-71$.

() Lee and Daoud; Licensee Bentham. Open.

This is an open access article licensed under the terms of the Creative Commons Attribution Non-Commercial License (http://creativecommons.org/licenses/ by-nc/3.0/) which permits unrestricted, non-commercial use, distribution and reproduction in any medium, provided the work is properly cited. 